\title{
Chebyshev Approximations for the Coulomb Phase Shift*
}

\author{
By W. J. Cody and K. E. Hillstrom
}

\begin{abstract}
This note presents nearly-best rational approximations for the Coulomb phase shift $\sigma_{0}(\eta)=\arg \Gamma(1+i \eta)$. Maximal relative errors range down to between $4.24 \times 10^{-10}$ and $1.09 \times 10^{-20}$. The nontrivial zero of $\sigma_{0}(\eta)$ is also given.
\end{abstract}

1. Introduction. The Coulomb wave functions $F_{L}(\eta, \rho)$ and $G_{L}(\eta, \rho)$ are the two real, linearly independent solutions of the second-order differential equation

$$
d^{2} y / d \rho^{2}+\left[1-2 \eta / \rho-L(L+1) / \rho^{2}\right] y=0
$$

that have the asymptotic behavior for large $\rho$

and

$$
F_{L}(\eta, \rho) \sim \sin \left(\rho-\eta \ln 2 \rho-\frac{L}{2} \pi+\sigma_{L}(\eta)\right)
$$

$$
G_{L}(\eta, \rho) \sim \cos \left(\rho-\eta \ln 2 \rho-\frac{L}{2} \pi+\sigma_{L}(\eta)\right)
$$

where the quantity

$$
\sigma_{L}(\eta)=\arg \Gamma(L+1+i \eta)=\operatorname{Im}\{\ln \Gamma(L+1+i \eta)\}
$$

is the Coulomb phase shift [1]. These Coulomb functions and phase shifts are frequently of importance in quantum-mechanical scattering problems.

At present, satisfactory techniques exist for calculation of the $F_{L}(\eta, \rho)$ [2], [3], but the techniques for calculating the $G_{L}(\eta, \rho)$ and the $\sigma_{L}(\eta)$ [4], [5] are slow and not too accurate. In particular, calculation of the $\sigma_{L}(\eta)$ is generally based on the asymptotic expansion of $\ln \Gamma(z)$. Using the first three terms in this expansion, we find

$$
\begin{aligned}
\sigma_{L-1}(\eta) \simeq & -\eta+\frac{\eta}{2} \ln \left(L^{2}+\eta^{2}\right)+\frac{2 L-1}{2} \arctan (\eta / L) \\
& +\frac{-\eta}{12\left(L^{2}+\eta^{2}\right)}\left[1+\frac{\eta^{2}-3 L^{2}}{30\left(L^{2}+\eta^{2}\right)^{2}}+\frac{\eta^{4}-10 L^{2} \eta^{2}+5 L^{4}}{105\left(L^{2}+\eta^{2}\right)^{4}}\right] .
\end{aligned}
$$

Lutz and Karvelis [4] use this approximation for $L=4$, and then calculate $\sigma_{0}(\eta)$, as well as $\sigma_{L}(\eta)$ for other $L$, from the relation

$$
\sigma_{0}(\eta)=\sigma_{L}(\eta)-\sum_{j=1}^{L} \arctan (\eta / j) .
$$

Received November 5, 1969.

AMS Subject Classifications. Primary 6520, 6525; Secondary 3315, 3330, 4140.

Key Words and Phrases. Rational Chebyshev approximations, Coulomb phase shift, complex gamma function.

* Work performed under the auspices of the U. S. Atomic Energy Commission.

Copyright @ 1971, American Mathematical Society 


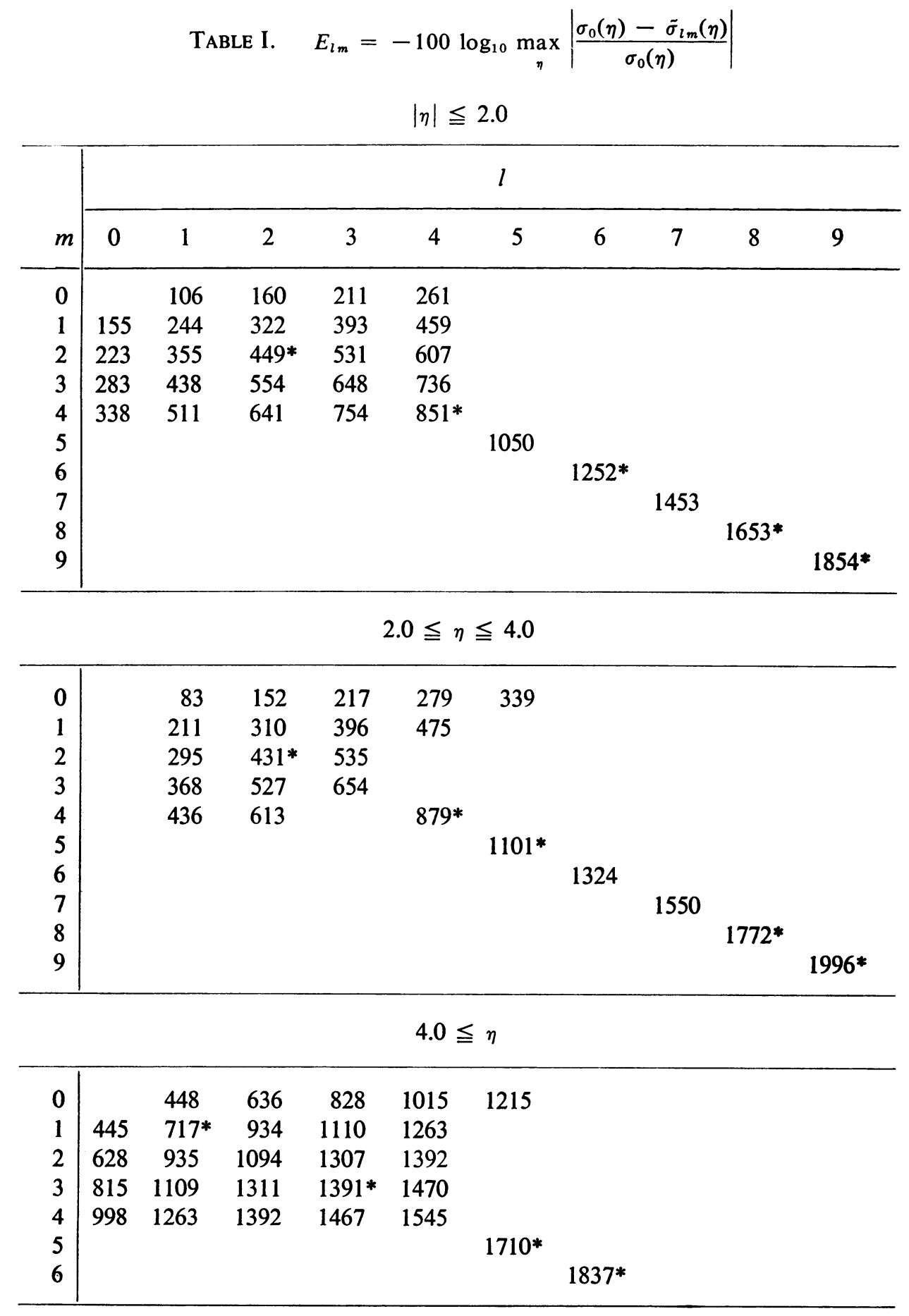

* Coefficients for these approximations only are given in Tables II-IV. 
In this note we present rational Chebyshev approximations that allow rapid, direct evaluation of $\sigma_{0}(\eta)$ for up to 20 significant decimal places. Equation (1.2) can then be used to find $\sigma_{L}(\eta)$ for other $L$. In addition, the nontrivial zero of $\sigma_{0}(\eta)$ is presented to 22 decimal places.

2. Generation of the Approximations. The forms of the approximations and the values of $\eta$ for which they are used are

$$
\begin{array}{rlrl}
\sigma_{0}(\eta) & \simeq \eta\left(\eta^{2}-\eta_{0}^{2}\right) R_{l m}\left(\eta^{2}\right), & 0 \leqq \eta \leqq 2.0, \\
& \simeq \eta R_{l m}\left(\eta^{2}\right), & 2.0 \leqq \eta \leqq 4.0, \\
& \simeq \frac{\arctan (\eta)}{2}+\eta\left[\frac{\ln \left(1+\eta^{2}\right)}{2}+R_{l m}\left(1 / \eta^{2}\right)\right], & & 4.0 \leqq \eta,
\end{array}
$$

where

$$
\eta_{0}=1.8055470716051069198764
$$

is the positive nontrivial zero of $\sigma_{0}(\eta)$, and the $R_{l m}(z)$ are rational functions of degree $l$ in the numerator and $m$ in the denominator.

Standard versions of the Remes algorithm for rational Chebyshev approximation [6], [7] were used to generate the rational approximations. Function values were computed as needed using various methcds, depending on the value of $\eta$. For small $\eta$, the ccmputation was based on the series expansion

(2.1) $\ln \Gamma(1+z)=-\ln (1+z)+z(1-\gamma)+\sum_{n=2}^{\infty}(-1)^{n}[\zeta(n)-1] \frac{z^{n}}{n},|z|<2$.

The power series for $\sigma_{0}(\eta)$ derived from (2.1) was transformed into a rapidly convergent and computationally stable continued fraction. Necessary values of $\zeta(n)-1$ were computed to an accuracy of $25 \mathrm{~S}$ with $40 \mathrm{~S}$ arithmetic.

For $\left|\eta-\eta_{0}\right|<.0005$, a Taylor's series expansion about $\eta_{0}$ was used. For all other values of $\eta, \sigma_{0}(\eta)$ was computed by first using the well-known convergent continued fraction to evaluate $\ln \Gamma(5+i \eta)$, and then recurrence (1.2). Newton's method was used to determine $\eta_{0}$.

All computations were carried out on a CDC-3600 computer in $25 \mathrm{~S}$ arithmetic. By comparing the various methods for computing $\sigma_{0}(\eta)$ against one another, the accuracy of the master routines was verified to at least $22 \mathrm{~S}$.

3. Results. Table I lists the values of

$$
E_{l m}=-100 \log _{10} \max _{\eta}\left|\frac{\sigma_{0}(\eta)-\tilde{\sigma}_{l m}(\eta)}{\sigma_{0}(\eta)}\right|,
$$

(here $\tilde{\sigma}_{l m}(\eta)$ denotes the appropriate approximation and the maximum is taken over the appropriate interval) for the initial segments of the various $L_{\infty}$ Walsh arrays. Tables II-IV present coefficients for selected approximations along the main diagonals of these arrays.

All coefficients are given to an accuracy slightly greater than that justified by the maximal errors, but reasonable additional rounding should not seriously affect 
TABLE II. $\quad \sigma_{0}(\eta) \simeq \sigma_{n n}(\eta)=\eta\left(\eta^{2}-\eta_{0}^{2}\right) \sum_{i=0}^{n} p_{i} \eta^{2 i} / \sum_{j=0}^{n} q_{i} \eta^{2 i} \quad|\eta| \leqq 2.0$

\begin{tabular}{|c|c|c|c|c|c|c|c|}
\hline \multirow{4}{*}{$\frac{n}{2}$} & $j$ & \multicolumn{3}{|c|}{$p_{i}$} & \multicolumn{3}{|c|}{$q_{i}$} \\
\hline & 0 & 1.468975 & & $(00)$ & 8.296754 & & (a) \\
\hline & 1 & 7.368587 & & $(-01)$ & 7.368168 & & $(00$ \\
\hline & 2 & 1.836788 & & $(-02)$ & 1.000000 & & (o) \\
\hline 4 & 0 & 2.8940039291 & & $(01)$ & 1.6344763441 & & $(0$ \\
\hline & 1 & 3.8279775977 & & $(01)$ & 2.7951966647 & & (02) \\
\hline & 2 & 1.3640064026 & & $(01)$ & 1.4602879586 & & \\
\hline & 3 & 1.1961484185 & & $(00)$ & 2.4851350161 & & $(0$ \\
\hline & 4 & 7.1411704272 & & $(-03)$ & 1.0000000000 & & \\
\hline 6 & 0 & $7.26231 \quad 75420$ & 0794 & $(02)$ & 4.1016137088 & 4059 & $(03)$ \\
\hline & 1 & 1.5482208152 & 6481 & $(03)$ & 1.0333098198 & 2759 & $(0$ \\
\hline & 2 & 1.1488420205 & 4676 & ( 03$)$ & $9.50548 \quad 18490$ & 6670 & $(0$ \\
\hline & 3 & 3.5487861514 & 8409 & $(02)$ & 3.9234452839 & 1116 & $(0$ \\
\hline & 4 & 4.2851543392 & 7621 & $(01)$ & 7.2315775777 & 89 & $(02$ \\
\hline & 5 & 1.5118553281 & 8749 & $(00)$ & 5.2081764819 & 7408 & $(0)$ \\
\hline & 6 & 3.7842131532 & 1365 & $(-03)$ & 1.0000000000 & 0000 & \\
\hline 8 & 0 & 2.0106142480 & 06387007 & $(04)$ & 5552707 & 66994359 & (05) \\
\hline & 1 & 5.9109915291 & 67068016 & $(04)$ & 3.7783496966 & 76579556 & $(0$ \\
\hline & 2 & 6.7266308978 & 48372087 & $(04)$ & 98426839 & $98281 \quad 1$ & $(0$ \\
\hline & 3 & 3.7529923373 & 45015120 & $(04)$ & 3.3439360399 & 70151738 & $(0$ \\
\hline & 4 & 1.0751139163 & 93479392 & $(04)$ & 1.2093893155 & 30544191 & $(0$ \\
\hline & 5 & 1.5170227985 & 59215518 & $(03)$ & 2.3293600087 & 64246542 & $(0$ \\
\hline & 6 & 9.2345741447 & $74635 \quad 177$ & $(01)$ & 2.2197274313 & 37920938 & $(0$ \\
\hline & 7 & $1.75100 \quad 14594$ & 87020923 & $(00)$ & 8.8738035740 & 41218697 & \\
\hline & 8 & $2.34438 \quad 18744$ & 24005593 & $(-03)$ & 1.0000000000 & 00000000 & \\
\hline 9 & 0 & 1.0887150490 & 479741168 & $(05)$ & 3478 & 7113 & $(0$ \\
\hline & 1 & 3.6470757308 & 1160914640 & ( 05$)$ & 0158 & 57080 & \\
\hline & 2 & $4.88801 \quad 47158$ & 2878013158 & $(05)$ & 3.5031084412 & 8424021934 & $(0$ \\
\hline & 3 & 3.3627573629 & 8197324009 & $(05)$ & 2.8119499028 & 6041080264 & $(0$ \\
\hline & 4 & 1.2689922627 & 7838479804 & $(05)$ & 1.2823644199 & 4358406742 & $(0$ \\
\hline & 5 & 2.6079554352 & 7084582682 & $(04)$ & 3.3520934871 & 1803753154 & \\
\hline & 6 & 2.7335248055 & 4497990544 & $(03)$ & 4.8431958024 & 7948701171 & \\
\hline & 7 & 1.2644754356 & 9902963184 & $(02)$ & 3.5487703900 & 6873206531 & $(C$ \\
\hline & 8 & 1.8544602212 & 5533909390 & $(00)$ & 1.1120720129 & 9804390166 & $(0$ \\
\hline & 9 & 1.9071621999 & 0037648146 & $(-03)$ & 1.0000000000 & 0000000000 & (0 \\
\hline
\end{tabular}


the accuracy of the approximations. All approximations listed were checked by comparing computations with the coefficients as they are presented here against computations with the master function routines.

With a little care, accurate computer subroutines that use these approximations can be written. For $0 \leqq \eta \leqq 2.0$, the computation of the factor $\eta^{2}-\eta_{0}^{2}$ is critical

TABLE III. $\quad \sigma_{0}(\eta) \simeq \tilde{\sigma}_{n n}(\eta)=\eta \sum_{j=0}^{n} p_{i} \eta^{2 i} / \sum_{j=0}^{n} q_{i} \eta^{2 i} \quad 2.0 \leqq \eta \leqq 4.0$

\begin{tabular}{|c|c|c|c|c|}
\hline \multirow{2}{*}{$\frac{n j}{20}$} & $p_{i}$ & \multicolumn{3}{|c|}{$q_{i}$} \\
\hline & -6.157559 & (01) & 1.189223 & $(02)$ \\
\hline 1 & 1.348358 & $(01)$ & 3.927495 & (01) \\
\hline 2 & 1.660751 & $(00)$ & 1.000000 & $(00)$ \\
\hline 40 & -1.3019963766 & (04) & 2.2700007374 & (04) \\
\hline 1 & -2.5846797911 & (03) & 1.9850722472 & (04) \\
\hline 2 & 1.4451643973 & (03) & 3.6894231707 & (03) \\
\hline 3 & 1.6840520098 & (02) & 1.5743762626 & $(02)$ \\
\hline 4 & 2.2385404975 & $(00)$ & 1.0000000000 & $(00)$ \\
\hline 50 & -1.9633277985787 & $(05)$ & 3.4068178321482 & (05) \\
\hline 1 & -8.5011405797202 & (04) & 3.8202031307722 & (05) \\
\hline 3 & 6.5546031287939 & (03) & $9.9836441488 \quad 102$ & (03) \\
\hline 4 & 3.1774547883836 & (02) & 2.4487049151213 & $(02)$ \\
\hline 5 & 2.4345650037542 & $(00)$ & 1.0000000000000 & $(00)$ \\
\hline 80 & -6.8561778344558127516 & $(08)$ & 1.1878300450036770748 & (09) \\
\hline 1 & -8.1383198338429411218 & (08) & 2.2343470745468131611 & (09) \\
\hline 2 & -1.8957959754898821821 & $(08)$ & 1.4530253317431556033 & (09) \\
\hline 3 & 5.2777180138860358173 & (07) & 4.1026369336998939991 & (08) \\
\hline 4 & 2.2441015897370915014 & $(07)$ & 5.3247562681404046468 & $(07)$ \\
\hline 5 & 2.3840547089679446349 & (06) & 3.1218892522014296487 & (06) \\
\hline 6 & 9.0106575392982150217 & $(04)$ & 7.5877858146972898291 & (04) \\
\hline 7 & 1.1078380003390400140 & $(03)$ & 6.2384723297519853161 & $(02)$ \\
\hline 8 & 2.8637139999878464927 & $(00)$ & 1.0000000000000000000 & $(00)$ \\
\hline 90 & -1.044100987526487618670 & (10) & 1.808868161493543887787 & (10) \\
\hline 1 & -1.508574107180079913696 & $(10)$ & 3.869142051704700267785 & (10) \\
\hline 2 & -5.582652833355901160542 & (09) & 3.003264575147162634046 & (10) \\
\hline 3 & 4.052529174369477275446 & (08) & 1.075554651494601843525 & (10) \\
\hline 4 & $5.4617122731 \quad 18594275192$ & (08) & 1.901298501823290694245 & (09) \\
\hline 5 & 9.510404403068169395714 & (07) & 1.665999832151229472632 & (08) \\
\hline 6 & 6.281126609997342119416 & (06) & 6.952188089169487375936 & (06) \\
\hline 7 & 1.651178048950518520416 & (05) & 1.253235080625688652718 & (05) \\
\hline 8 & $\begin{array}{llll}1.49882 & 4421329341285521\end{array}$ & (03) & 7.904420414560291396996 & $(02)$ \\
\hline 9 & 2.974686506595477984776 & $(00)$ & 1.000000000000000000000 & $(00)$ \\
\hline
\end{tabular}


TABLE IV. $\quad \sigma_{0}(\eta) \simeq \sigma_{n n}(\eta)=\frac{\arctan (\eta)}{2}+\eta\left[\frac{\ln \left(1+\eta^{2}\right)}{2}+\sum_{i=0}^{n} p_{i} \eta^{-2 i} / \sum_{i=0}^{n} q_{i} \eta^{-2 i}\right]$ $4.0 \leqq \eta$

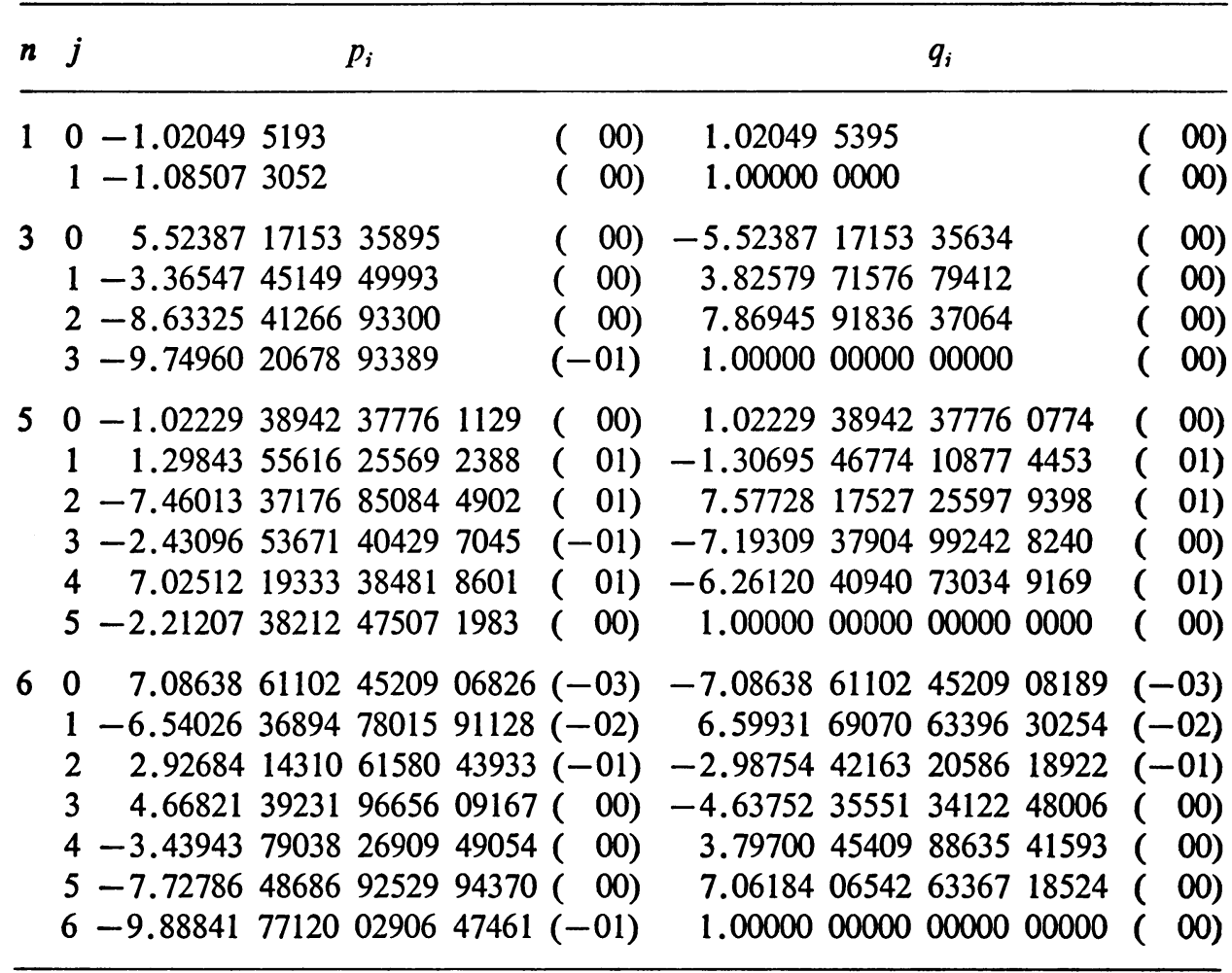

when $\eta \simeq \eta_{0}$ if one is to maintain essential machine precision in the function value. It is best to compute $\eta^{2}-\eta_{0}^{2}$ as $\left(\eta-\eta_{0}\right)\left(\eta+\eta_{0}\right)$, with the factor $\eta-\eta_{0}$ computed to higher than machine precision. This can be accomplished by representing $\eta_{0}$ in two parts, $\eta_{0} \equiv \eta_{1}+\eta_{2}$, such that the floating point exponent for $\eta_{2}$ is much less than that for $\eta_{1}$. To facilitate that representation, we give both the octal and hexadecimal representation of $\eta_{0}$ :

$$
\begin{aligned}
\eta_{0} & =1.6343412515767270471044565_{8} \\
& =1 . C E 385537 \mathrm{EE} \mathrm{B89C8} 92 \mathrm{EA}_{18} .
\end{aligned}
$$

Then $\left(\eta-\eta_{0}\right)$ may be computed accurately as $\left(\eta-\eta_{0}\right)=\left(\eta-\eta_{1}\right)-\eta_{2}$.

In addition, in at least one case the conditioning of an approximation in the interval $2.0 \leqq \eta \leqq 4.0$ was improved by evaluating it in the form

$$
\eta \sum_{i=0}^{n} p_{n-i} \eta^{-2 i} / \sum_{i=0}^{n} q_{n-i} \eta^{-2 i}
$$

by use of nested multiplication. 
If function values are desired for negative arguments, the relation

$$
\sigma_{0}(-\eta)=-\sigma_{0}(\eta)
$$

can be used.

Argonne National Laboratory

Argonne, Illinois 60439

1. M. Abramowitz, "Coulomb wave functions," Handbook of Mathematical Functions with Formulas, Graphs, and Mathematical Tables, M. Abramowitz \& I. A. Stegun (Editors), Nat. Bur. Standards Appl. Math. Series, 55, Superintendent of Documents, U.S. Government Printing Office, Washington, D.C., 1964, chap. 14, pp. 537-554. MR 29 \#4914.

2. W. GAUTSCHI, "Computational aspects of three-term recurrence relations," SIAM Rev., v. 9, 1967, pp. 24-82. MR 35 \#3927.

3. W. GAUTSCHI, "Algorithm 292, regular Coulomb wave functions," Comm. ACM, v. 9, 1966, pp. 793-795.

4. H. F. LUTZ \& M. D. Karvelis, "Numerical calculation of Coulomb wave functions for repulsive Coulomb fields," Nuclear Phys., v. 43, 1963, pp. 31-44.

5. J. H. GunN, "Algorithm 300, Coulomb wave functions," Comm. ACM, v. 10, 1967, pp. 244-245.

6. W. J. CODY, W. FrASER \& J. F. HART, "Rational Chebyshev approximation using linear equations," Numer. Math., v. 12, 1968, pp. 242-251.

7. H. Werner, J. Stoer \& W. Bommas, "Rational Chebyshev approximation," Numer. Math., v. 10, 1967, pp. 289-306. 